\title{
Promyelocytic leukemia nuclear body disruption as a treatment for EBV-associated nasopharyngeal carcinoma?
}

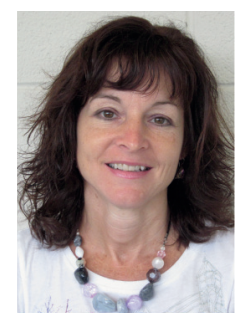

“...ATO in combination with ganciclovir resulted in the loss of $75 \%$ of the EBV-positive cells without affecting the EBV-negative cells. These results have important implications for treatment of EBV-positive NPC..."

\section{Lori Frappier}

Department of Molecular Genetics, University of Toronto, 1 Kings College Circle, Toronto M3A 2M1, Canada = lori.frappier@utoronto.ca

EBV is highly recognized as a causative agent in the development of several types of cancer, originating with its initial discovery in Burkitt's lymphoma tumor samples. Since then, EBV infection has been found to be strongly associated with several types of B-cell lymphomas as well as two types of epithelial cell tumors; nasopharyngeal carcinoma (NPC) and gastric carcinoma. Virtually all undifferentiated NPC tumors (the most common type) are monoclonal expansions of EBV-infected cells, whereas EBV infection is limited to approximately $10 \%$ of all gastric carcinomas, in particular those located at the top of the stomach [1,2].

\footnotetext{
"Recently, Sides et al. presented a new approach to induce the EBV Iytic cycle through the disruption of promyelocytic leukemia nuclear bodies."
}

EBV is a $\gamma$-herpes virus and, like all herpes viruses, has both latent and lytic (or productive) modes of infection. EBV most often results in latent infection and it is this form of infection that is found in EBV-associated tumors. EBV latent infection can induce cell proliferation and increase cell survival (often referred to as immortalization) through the expression of EBV nuclear antigens and latent membrane proteins. The ability of EBV latent infection to increase cell proliferation and survival likely accounts for its ability to promote the development of cancer. Different subsets of EBV latency proteins are expressed in different latency types. In NPC, only one EBV nuclear protein (EBNA1), one membrane protein (LMP2) and one secreted protein (BARF1) are consistently expressed, while LMP1 is sometimes detected [1]. In latent infection, EBV genomes are maintained as double-stranded circular molecules that replicate once per cell cycle and are evenly segregated to the daughter cells through tethering to the host chromosomes [3]. EBV latent infection can switch to lytic infection, although exactly how this switch is triggered is not clear. EBV lytic infection results in a cascade of expression of approximately 80 proteins, and amplification of the viral genome into linear DNA concatemers that are cleaved into unit length upon packaging in the viral capsids. The capsids ultimately acquire an envelope from the plasma membrane or post-Golgi vesicles and virions are released from the cell surface.

Lytic infection results in cell-cycle arrest and eventually in cell death. In addition, cells that are lytically infected with EBV are susceptible to the cytotoxic effects of ganciclovir, a nucleoside analog that is converted to its active form after it is phosphorylated by an EBV-encoded kinase that is only expressed in lytic infection. Therefore one strategy that has been explored for treating EBV-positive tumors is to induce the lytic cycle in the latently infected tumor cells and to treat with ganciclovir $[4,5]$. To this end, drugs that activate immediate early EBV gene expression through a variety of mechanisms have been used to reactivate EBV and to kill EBVpositive tumor cells in conjunction with ganciclovir. These include treatments that affect DNA methylation, histone acetylation, protein kinase signaling and activation of the B-cell receptor [6]. Recently, Sides et al. presented a new approach to induce the EBV lytic cycle through the disruption of promyelocytic leukemia (PML) nuclear bodies (NBs) [7]. PML NBs are nuclear foci that contain many proteins but depend on

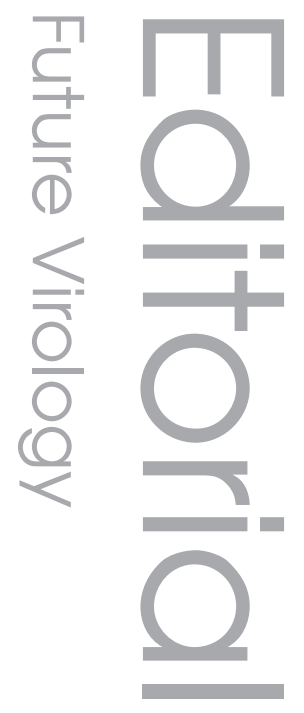

\section{Keywords}

m arsenic = Epstein-Barr virus = ganciclovir $=$ promyelocytic leukemia protein

\section{Future : Medicine ${ }_{\text {part of }}$}


PML proteins for their structural organization [8]. These NBs mediate several cellular processes, including apoptosis and DNA repair. PML NBs also suppress lytic infection of several viruses, including HSV-1 and CMV, and therefore the ability of specific viral proteins to disrupt PML NBs is important for efficient lytic infection [9]. PML NBs can also be disrupted by treating cells with arsenic trioxide (ATO), which has been used for some time as a treatment for acute PML [10]. ATO is effective in treating PML because these cells express an aberrant form of PML fused to the retinoic acid receptor- $\alpha$. Arsenic has been shown to interact with PML and trigger its SUMOylation, which results in the recruitment of the RNF4 E3 ubiquitin-ligase and PML polyubiquitylation and degradation $[11,12]$.

\section{“...the ATO/ganciclovir combination treatment provides a promising therapeutic approach at least for NPC and potentially for all EBV-positive cancers."}

Sides et al. examined the effect of ATO treatment on the reactivation of EBV from latent infection in lung adenocarcinoma (A549) and NPC (CNE1) cells [7]. All detectable PML NBs were lost from the A549 and CNE1 cells after treatment with $12.5 \mathrm{nM}$ ATO and $1 \mathrm{nM}$ ATO, respectively, and the PML loss was accompanied by an increase in the expression of EBV immediate-early and early proteins, suggesting that most of the cells had entered the lytic cycle. The CNE1 cells were found to be particularly sensitive to ATO, as the $1 \mathrm{nM}$ ATO found to efficiently induce PML loss and reactivation is considerably below the concentrations typically used to induce PML disruption in cell lines (usually $0.1-1 \mu \mathrm{M}$ ). It is also below the serum arsenic levels resulting from treatment of acute PML with US FDA-approved amounts of ATO. While it is not clear from these studies whether the ATO treatment induced the complete lytic cycle or an abortive lytic infection, this treatment was sufficient to sensitize the cells to ganciclovir. While ATO treatment alone had only a small effect on cell viability, ATO in combination with ganciclovir resulted in the loss of $75 \%$ of the EBV-positive cells without affecting the EBV-negative cells. These results have important implications for treatment of EBV-positive NPC, especially considering that both drugs are effective, at least in tissue culture, at concentrations already approved for use in humans.
However, several questions remain to be addressed, one of which is whether other NPC cell lines containing EBV are similarly sensitive to lytic induction by ATO. In particular, it would be interesting to examine the only naturally occurring EBV-positive NPC cell line, C666-1. Unlike the EBV-infected CNE1 cells used in the Sides et al. study, C666-1 and most NPC tumor cells do not express LMP1. Similarly LMP1 is not expressed in EBV-positive gastric carcinomas. This could influence the ATO effect since Sides et al. found that LMP1 expression induced PML NB and protein levels. In contrast, C666-1 and an EBV-positive gastric carcinoma cell line (AGS-EBV) both have very low levels of PML NBs and proteins due to the action of EBNA1, which induces the degradation of PML proteins in these cells $[13,14]$. This appears to extend to tumors as EBV-positive gastric carcinoma biopsies have less PML staining than their EBV-negative counterparts [14]. However, our recent data indicates that PML loss promotes the reactivation of EBV in AGS-EBV, as observed by PML silencing, and therefore it seems likely that ATO would induce the EBV lytic cycle in these cells and in EBVpositive carcinomas in general [SIVACHANDRAN N, WANG X, Frappier L. Functions of the Epstein-Barr Virus LATENCY PROTEIN, EBNA1, IN THE VIRAL REACTIVATION AND iytic infection (2011), SUbmitted]. Another clinically important question is whether ATO in combination with ganciclovir would effectively kill EBV-positive B-cell lymphomas. Inducing EBV reactivation in $\mathrm{B}$-cells is typically more difficult than in epithelial cells since B-cells are less permissive to lytic infection. Hence, treatments that induce the lytic cycle in EBV-positive epithelial cell lines do not necessarily do so in EBV-positive B-cell lines. It will be interesting to determine if ATO has a more widespread effect on EBV reactivation. Regardless, the ATO/ganciclovir combination treatment provides a promising therapeutic approach at least for NPC and potentially for all EBV-positive cancers.

\footnotetext{
Financial \& competing interests disclosure

The author has no relevant affiliations or financial involvement with any organization or entity with a financial interest in or financial conflict with the subject matter or materials discussed in the manuscript. This includes employment, consultancies, honoraria, stock ownership or options, expert testimony, grants or patents received or pending, or royalties.

No writing assistance was utilized in the production of this manuscript.
} 


\section{References}

1. Tao Q, Chan AT. Nasopharyngeal carcinoma: molecular pathogenesis and therapeutic developments. Expert Rev. Mol. Med. 9, 1-24 (2007).

2. Fukayama M. Epstein-Barr virus and gastric carcinoma. Pathol. Int. 60, 337-350 (2010).

3. Frappier L. EBNA1 in viral DNA replication and persistence. In: Epstein-Barr Virus: Latency and Transformation. Robertson ES (Ed.). Caister Academic Press, UK, 37-59 (2010).

4. Feng WH, Israel B, Raab-Traub N, Busson P, Kenney SC. Chemotherapy induces lytic EBV replication and confers ganciclovir susceptibility to EBV-positive epithelial cell tumors. Cancer Res. 62, 1920-1926 (2002).

5. Feng WH, Hong G, Delecluse HJ, Kenney SC. Lytic induction therapy for Epstein-Barr virus-positive B-cell lymphomas. J. Virol. 78, 1893-1902 (2004).
6. Amon W, Farrell PJ. Reactivation of EpsteinBarr virus from latency. Rev. Med. Virol. 15, 149-156 (2005).

7. Sides MD, Block GJ, Shan B et al. Arsenic mediated disruption of promyelocytic leukemia protein nuclear bodies induces ganciclovir susceptibility in Epstein-Barr positive epithelial cells. Virology 416, 86-97 (2011).

8. Bernardi R, Pandolfi PP. Structure, dynamics and functions of promyelocytic leukaemia nuclear bodies. Nat. Rev. Mol. Cell Biol. 8, 1006-1016 (2007).

9. Geoffroy MC, Chelbi-Alix MK. Role of promyelocytic leukemia protein in host antiviral defense. J. Interferon Cytokine Res. 31, 145-158 (2011).

10. Lengfelder E, Hofmann WK, Nowak D. Impact of arsenic trioxide in the treatment of acute promyelocytic leukemia. Leukemia doi:10.1038/leu.2011.245 (2011) (Epub ahead of print).
11. Lallemand-Breitenbach V, Jeanne M, Benhenda $S$ et al. Arsenic degrades PML or PML-RARalpha through a SUMO-triggered RNF4/ubiquitin-mediated pathway. Nat. Cell Biol. 10, 547-555 (2008).

12. Tatham MH, Geoffroy MC, Shen L et al. RNF4 is a poly-SUMO-specific E3 ubiquitin ligase required for arsenic-induced PML degradation. Nat. Cell Biol. 10, 538-546 (2008).

13. Sivachandran N, Sarkari F, Frappier L. Epstein-Barr nuclear antigen 1 contributes to nasopharyngeal carcinoma through disruption of PML nuclear bodies. PLoS Pathog. 4, E1000170 (2008).

14. Sivachandran N, Dawson CW, Young LS, Liu F, Middeldorp J, Frappier L. Contributions of the Epstein-Barr virus EBNA1 protein to gastric carcinoma. J. Virol. doi:JVI.0562311v1-JVI.05623-11 (2011) (Epub ahead of print). 